\title{
Author Correction: Nuclear-mitochondrial DNA segments resemble paternally inherited mitochondrial DNA in humans
}

\author{
Wei Wei $\mathbb{B}$, Alistair T. Pagnamenta (D), Nicholas Gleadall, Alba Sanchis-Juan, Jonathan Stephens, \\ John Broxholme, Salih Tuna (1), Christopher A. Odhams, Genomics England Research Consortium, NIHR \\ BioResource, Carl Fratter (1), Ernest Turro (1), Mark J. Caulfield (1), Jenny C. Taylor, Shamima Rahman (1) \& \\ Patrick F. Chinnery
}

Correction to: Nature Communications https://doi.org/10.1038/s41467-020-15336-3, published online 8 April 2020.

The original version of this Article contained an error in an equation in the Methods in the section "Estimating the number of mtDNA fragments within each NUMT". The equation for calculating the number of mtDNA fragments within each nuclear-mitochondrial DNA segment and its explanation incorrectly read:

The number of copies of mtDNA-derived fragments (Nmt) within the same NUMT was estimated as:

$$
\mathrm{Nmt}=\frac{\text { DPnumt } \div 2}{\text { DPnumt } \div 2+\text { Altmt }}
$$

where DPnumt is the average depth of the NUMT sequence surrounding region where discordant reads aligned on the nuclear genome; and Altmt is the number of reads supported alternative allele from the informative variants within the mixed haplotype. If the $\mathrm{AF}>50 \%$, Altmt $=\mathrm{DPmt}-$ Altmt'. DPmt is the depth of the informative variant, Altmt' is the initial number of reads supported alternative allele.

The correct version of this paragraph is:

The number of copies of mtDNA-derived fragments (Nmt) within the same NUMT was estimated as:

$$
N m t=\frac{\text { Altmt }}{\text { DPadjnumt } \div 2}
$$

where DPadjnumt is the average depth of the nuclear genome sequencing flanking the NUMT (derived from both complementary chromosomes); and Altmt is the number of reads supporting the alternative allele from the informative variants within the mixed haplotype. If the $\mathrm{AF}>50 \%$, Altmt $=\mathrm{DPmtvar}-$ Altmt'. DPmtvar is the depth of the informative variant, Altmt' is the initial number of reads supported alternative allele.

We confirm that the correct equation was used in our analyses, and that this correction does not change our results.

This has now been corrected in the PDF and HTML versions of the Article.

Published online: 22 July 2020 
(c) Open Access This article is licensed under a Creative Commons Attribution 4.0 International License, which permits use, sharing, adaptation, distribution and reproduction in any medium or format, as long as you give appropriate credit to the original author(s) and the source, provide a link to the Creative Commons license, and indicate if changes were made. The images or other third party material in this article are included in the article's Creative Commons license, unless indicated otherwise in a credit line to the material. If material is not included in the article's Creative Commons license and your intended use is not permitted by statutory regulation or exceeds the permitted use, you will need to obtain permission directly from the copyright holder. To view a copy of this license, visit http://creativecommons.org/licenses/by/4.0/.

(C) The Author(s) 2020 